\title{
Reduciendo la ideación suicida en adolescentes con terapia de perspectiva temporal. Un estudio experimental
}

\author{
Cristián Oyanadel; Héctor Carrasco-Correa; Javier Latorre-Nanjarí; \\ Wenceslao Peñate-Castro; Camilo Sepúlveda-Queipul
}

Cómo citar este artículo:

Oyanadel, C., Carrasco-Correa, H., Latorre-Nanjarí, J., Peñate-Castro, W., \& Sepúlveda-Queipul, C. (2021). Reduciendo la ideación suicida en adolescentes con terapia de perspectiva temporal. Un estudio experimental. Acta Colombiana de Psicología, 24(1), 63-71. https://www.doi.org/10.14718/ACP.2021.24.1.6

Recibido, junio 19/2019; Concepto de evaluación, marzo 03/2019; Aceptado, mayo 07/2020

\author{
Cristián Oyanadel ${ }^{1}$ \\ ORCID: https://orcid.org/0000-0001-8423-6982 \\ Universidad de Concepción, Concepción, Chile. \\ Héctor Carrasco-Correa \\ ORCID: https://orcid.org/0000-0003-2178-9891 \\ Universidad Católica del Norte, Coquimbo, Chile. \\ Javier Latorre-Nanjarí \\ ORCID: https://orcid.org/0000-0001-7171-5802 \\ Universidad de La Serena, La Serena, Chile. \\ Wenceslao Peñate-Castro \\ ORCID: https://orcid.org/0000-0001-9168-9920 \\ Universidad de La Laguna, La Laguna, España. \\ Camilo Sepúlveda-Queipul \\ ORCID: https://orcid.org/0000-0002-2393-2113 \\ Hospital de La Serena, La Serena, Chile.
}

\begin{abstract}
Resumen
El suicidio es una de las principales causas de muerte en el mundo, problema que en los últimos años ha venido en aumento sostenido para la población adolescente. En particular, la ideación suicida se ha relacionado fuertemente con el intento suicida, un predictor directo del suicidio; sin embargo, se encuentran escasas estrategias para disminuir la ideación suicida diseñadas principalmente para la depresión. Considerando las posibilidades terapéuticas de la teoría de Zimbardo sobre la perspectiva temporal equilibrada, en el presente estudio se diseñó una intervención breve basada en la perspectiva del tiempo para adolescentes con el objetivo de evaluar el efecto de una intervención de perspectiva temporal en la modificación de indicadores de ideación y conducta suicida en adolescentes. De este modo, se realizó un estudio experimental, donde 82 participantes de 15 a 19 años fueron asignados a una intervención experimental o a un grupo control. Los participantes del grupo experimental $(n=43)$ participaron durante 8 sesiones del programa de intervención de perspectiva del tiempo denominado "Serenamente". Las mediciones pre-post se realizaron sobre las variables de ideación suicida (Okasha), el perfil temporal (ZTPI) y la calidad de vida (SF-36). Como resultados, se encontró que la intervención reduce la ideación suicida en un $41 \%$; y que, posiblemente, mejora la salud física de quienes reciben este tipo de tratamiento. Los resultados son un avance promisorio con fines preventivos en contextos educativos y de salud.

Palabras clave: adolescente, ideación suicida, perspectiva temporal, estudio experimental, calidad de vida.
\end{abstract}

1 Barrio Universitario s/n, Fac. Cs. Sociales, Universidad de Concepción, Concepción, Chile. Correo electrónico: coyanadel@udec.cl Agradecimientos: este artículo ha sido realizado gracias al financiamiento de los Ministerios de Salud y de Educación de Chile, Proyectos: ANID-FONIS ref. SA13I20188, y los proyectos de cooperación internacional de ANID PAI80160045 y MEC80180087. 


\title{
Reducing suicidal ideation in adolescents with time perspective therapy. An experimental study
}

\begin{abstract}
Suicide is one of the leading causes of death in the world, a problem that has been steadily increasing in recent years for the adolescent population. In particular, suicidal ideation has been strongly associated with suicide attempt, a direct predictor of suicide; however, few strategies to decrease suicidal ideation designed primarily for depression are found. Considering the therapeutic possibilities of Zimbardo's theory on balanced time perspective, in the present study a brief intervention for adolescents based on that theory was designed with the aim of evaluating the effect of a time perspective intervention on the modification of indicators of suicidal ideation and behavior in adolescents. Thus, an experimental study was conducted, where 82 participants aged 15 to 19 were assigned to either an experimental intervention or a control group. The participants of the experimental group $(n=43)$ engaged in eight sessions of the time perspective intervention program called "Serenity". Pre-post measurements were made on the variables of suicidal ideation (Okasha), time profile (ZTPI) and quality of life (SF36). Results show that the intervention reduces suicidal ideation by $41 \%$; and that, possibly, it improves the physical health of those who receive this type of treatment. The results are a promising advance for preventive purposes in educational and health contexts.

Keywords: adolescent, suicide ideation, time perspective, experimental study, quality of life.
\end{abstract}

\section{Introducción}

El suicidio adolescente es un problema de salud que cada año sorprende por el aumento de su magnitud (OMS, 2014). Según el Ministerio de Salud de Chile (minsal, 2013), aproximadamente un millón de personas se suicidan en el mundo cada año, y en muchos países el suicidio es una de las cinco primeras causas de mortalidad en la franja de edad entre los 15 y los 19 años.

Como se ha encontrado en diversas investigaciones, la conducta suicida puede ser influida por aspectos culturales (Fernandes-Eloi \& Costa Lourenço, 2019); sin embargo, esta trasciende diferentes configuraciones sociales. Por ejemplo, se ha encontrado que el suicidio es la primera causa de muerte de adolescentes en Japón, y que está en los primeros lugares en otros tantos países (Kinoshita et al., 2012).

En particular, la ideación suicida se ha entendido como pensamientos cuya utilidad es buscar la propia muerte, que varían desde pensamientos de inutilidad a deseos de morir, con o sin planificación o método (MINSAL, 2013). Asimismo, la presencia de ideación suicida se considera un signo de alerta que pone en evidencia la vulnerabilidad de la persona al suicidio (Bahamón-Muñetón \& Alarcón-Vásquez, 2018), lo que hace que sea un fenómeno difícil de abordar, ya que da origen a tipologías distintas entre las personas para las que se vuelve un problema (American Psychiatric Association [APA], 2003; Turecki \& Brent, 2016).

Ahora, si bien la ideación suicida no se ha establecido como un predictor directo del suicidio, esta sí predice el intento suicida, el cual sí se considera un predictor potente del suicidio. De esta manera, la complejidad de la materia es tal que dicha conducta fue tipificada con cualidades específicas en la clasificación del DSM-5 (Asociación Americana de Psiquiatría [APA], 2013).

En América Latina, este problema de salud pública es uno de los más preocupantes por su alta prevalencia. Por ejemplo, en Colombia, el suicidio se encuentra entre las tres primeras causas de muerte en las personas de 15 a 44 años (Avendaño-Prieto et al., 2020), grupo en el cual los adolescentes entre 12 y 20 años presentan mayor alerta, pues representan el $43 \%$ de riesgo de suicidio (MartínezBaquero et al., 2017). Por otra parte, se considera que Chile es uno de los países con mayor ideación suicida, pues llega al $59 \%$ de prevalencia en el grupo de 14 a 19 años (VeraRomero \& Días-Vélez, 2012); porcentaje bastante alto, y que probablemente se relaciona con el aumento del $60 \%$ de la tasa de suicidio desde 1999 (Ventura-Juncá et al., 2010). De hecho, según el Plan Nacional de Salud Mental (2017-2025) de Chile, la tasa de suicidios en el grupo de adolescentes de entre 10 y 14 años aumentó de 0.96 en el año 2000 a 2.15 por cada 100000 habitantes el año 2008; mientras que en el grupo de 15 a 19 años la tasa de suicidio aumentó de 8.6 a 11.4 por cada 100000 habitantes en el mismo periodo (MINSAL, 2017).

Ahora bien, la adolescencia corresponde a una etapa de la vida particularmente sensible, ya que los adolescentes se encuentran expuestos a múltiples dificultades, sean familiares o escolares, que generan malestar o síntomas emocionales. Debido a algunas circunstancias en estas 
áreas, el nivel de ideación suicida puede aumentar, por ejemplo, si hay presencia de acoso escolar más allá de otras variables - como los síntomas depresivos de base(Ceballos-Ospino et al., 2019).

Teniendo lo anterior en cuenta, se han intentado establecer distintos programas preventivos para la desesperanza e ideación suicida; sin embargo, no se han desarrollado programas psicoterapéuticos específicos y eficaces, ya que los que se realizan suelen estar en una etapa inicial, y en un gran número son adaptaciones de terapias para la depresión que no tienen resultados concluyentes (Cuijpers et al., 2013). Ahora, aunque existe evidencia de experiencias en programas creados para tratar pacientes ingresados a unidades de hospitalización por intento suicida (Ghahramanlou-Holloway et al., 2012; LaCroix et al., 2018) o como prevención con programas en línea con adultos en población de riesgo (Guille et al., 2015; Pospos et al., 2018), como denominador común, son programas que cuentan con evidencia, pero no están diseñados para adolescentes y se basan solo en estrategias de terapias cognitivo-conductuales.

En esta búsqueda de tratamientos, la orientación o perspectiva temporal aparece como uno de los aspectos que se han indagado en relación con el suicidio. Por ejemplo, hace 50 años, una investigación planteaba que el suicidio puede deberse a un desequilibrio en la orientación temporal, emergente de una orientación negativa hacia el pasado, con marcados eventos negativos, sumados a una desconexión con el futuro (Yufit et al., 1970). Años más tarde, la hipótesis sobre la relación entre el suicidio adolescente y el desequilibrio temporal sería demostrada; no obstante, la idea de desconexión con el futuro sería reemplazada por el concepto de "presente fatalista" (Laghi et al., 2009).

Específicamente, la dimensión "presente fatalista" está relacionada con el concepto de desesperanza y falta de proyecciones, y es una de las cinco dimensiones temporales descritas por Zimbardo y Boyd (2009) en una teoría que la distingue del "pasado positivo y negativo", el "presente hedonista y fatalista", el "futuro trascendental" y el "futuro orientado a metas". En particular, estas dimensiones se suelen evaluar con el inventario Zimbardo Time Perspective Inventory (ZTPI), validado por Zimbardo y Boyd (1999), un instrumento que cuenta con una adaptación en Chile realizada por Oyanadel, Buela-Casal y Pérez-Fortis (2014).

Ahora bien, la perspectiva temporal ha sido evaluada en muchos estudios relacionados con la salud. Por ejemplo, Bourdon et al. (2020) demostraron que el estrés crónico multisistémico —-medido por 23 biomarcadores neuroendocrinos, inmunes, metabólicos y cardiovasculares — se vincula con la perspectiva del tiempo; mientras que Papastamatelou y Unger (2018), en un estudio alemán, muestran cómo el pasado negativo y el presente fatalista se identifican como factores significativos que aumentan el agotamiento emocional, mientras que el presente hedonista lo reduce.

De hecho, la medición de las distintas dimensiones temporales permite evaluar el perfil temporal de las personas. Así, un perfil temporal equilibrado, es decir, un balance entre las distintas dimensiones temporales, con elementos negativos del pasado y fatalistas del presente más bajos, se ha relacionado con mejores indicadores de bienestar y salud en diferentes países, como Inglaterra, Rusia (Boniwell et al., 2010), España y Chile (Oyanadel \& Buela-Casal, 2011; 2017). Específicamente, se han relacionado perfiles temporales a la dinámica del cortisol, con lo cual se evidencia la relación entre la perspectiva del tiempo y la modulación del estrés (Olivera-Figueroa et al., 2015). Asimismo, otros estudios muestran evidencia de la relación entre perfiles desadaptativos y el estrés (Papastamatelou et al., 2015).

Con el fin de modificar el perfil temporal de las personas, Sword et al. (2014) y Zimbardo et al. (2012) diseñaron la Terapia de la Perspectiva del Tiempo, la cual, inicialmente, trabajó con un programa para casos de estrés postraumático que tuvo buenos resultados (Sword et al., 2015). En ella, se incluye la evaluación de las tres dimensiones temporales —pasado, presente y futuro- de la persona, y se interviene por medio de ejercicios cognitivos y de meditación. Partiendo de esta perspectiva, otros autores se han focalizado en el entrenamiento en orientación al futuro con pacientes suicidas (Van Beek et al., 2009).

En Chile, en particular, hay evidencia(Oyanadel, BuelaCasal, Araya et al., 2014) que muestra cómo se puede modificar el perfil temporal por medio de la ejecución de ejercicios de meditación por mindfulness - conciencia o atención plena en español-, un concepto que se ha relacionado con la perspectiva temporal (Seema \& Sircova, 2013), y que evidencia resultados positivos de salud en distintos contextos clínicos (Didonna, 2011).

Respecto al mindfulness, con esta técnica se busca dirigir la atención al presente como uno de sus objetivos principales, y se considera que representa un concepto que tiene implicaciones intrínsecas de perspectiva temporal (Olivera-Figueroa et al., 2016). Según Rönnlund et al. (2019), la implementación de esta forma de intervención ha evidenciado, entre otros aspectos, que un mayor nivel de mindfulness promueve una perspectiva temporal más equilibrada.

Con estos antecedentes, el principal objetivo del presente estudio fue evaluar el efecto de una intervención grupal de perspectiva temporal en la modificación de indicadores de ideación y conducta suicida en adolescentes; y, específicamente, se buscó: (a) medir los cambios en 
ideación y conducta suicida en los adolescentes después del tratamiento; (b) evaluar si existen cambios en la calidad de vida relacionada a la salud en adolescentes después del tratamiento; y (c) comprobar si el perfil temporal de los participantes es modificable hacia un perfil más equilibrado.

\section{Método}

Tipo de estudio y diseño

Se diseñó un estudio experimental [1:1] (Montero \& León, 2007) de dos brazos, con grupos paralelos, una condición control en espera y una condición experimental dada por el programa. La variable principal de resultado fue la ideación suicida, medida con la puntuación en la adaptación chilena de la Escala de suicidalidad de Okasha (Salvo et al., 2009). Como variables secundarias, se evaluaron los niveles de calidad de vida, medidos con la versión chilena del cuestionario de salud SF-36 (Olivares, 2006), y el perfil temporal, medido con la versión chilena del ZTPI (Oyanadel \& Buela-Casal, 2014).

\section{Participantes}

Para el tamaño de la muestra se calculó la formula de Rosner; y los parámetros que se consideraron fueron los siguientes:

Poder estadístico $1-\beta=0.90(z=1.28)$, Error tipo I $\alpha=.05(z=1.96)$.

$$
n=\frac{p_{0}\left(1-p_{0}\right)\left(z_{1-\alpha / 2+z 1-\beta} \sqrt{\frac{p_{1}\left(1-p_{1}\right)}{p_{0}\left(1-p_{0}\right)}}\right)^{2}}{\left(p_{1}-p_{0}\right)^{2}}
$$

$\mathrm{H}_{0}: \mathrm{P}_{0}=0.60-$ prevalencia de adolescentes entre 14 y 19 años con ideación suicida-

$\mathrm{H}_{1}: \mathrm{P}_{1}=0.30-\mathrm{el}$ objetivo en estudios similares es reducir a $30 \%$ la prevalencia de ideación suicida en los participantes del grupo experimental-.

Teniendo esto en cuenta, se consideró que, al inicio, 6 de cada 10 participantes presentarían síntomas de ideación suicida; y que después de la intervención solo 3 de cada 10 participantes en el grupo experimental presentarían dichos síntomas. Esto implicaba que se necesitaban 27 participantes por grupo. De esta manera, y considerando una deserción de $50 \%$, se postuló como necesaria la participación de 41 sujetos por grupo - 82 participantes en total- para poner a prueba las hipótesis.

Contando con la aprobación del comité de ética clínico del Ministerio de Salud de Chile, zona norte, y en concordancia con la declaración de Helsinski, los participantes firmaron el consentimiento informado junto a su familiar responsable. Para la selección de la muestra, se realizó una pesquisa inicial de 264 adolescentes de entre 15 y 19 años de la provincia de Coquimbo, Chile, al ser este uno de los grupos de edad identificados como de riesgo según los registros de población bajo control adolescente que se cuantifican en dos grupos definidos (10-14 y 15-19).

Tras esta primera selección, se llegó a establecer 87 candidatos al programa, de los cuales finalmente 85 fueron asignados - al azar - al grupo experimental $(n=43)$ $\mathrm{y}$ al grupo control $(n=42)$. Debido a tres retiros, el grupo control se redujo a 39 participantes (véase Figura 1), y posteriormente a 38 en el postest.

El grupo control (79.5\% varones) tuvo una media de edad de 16.03 años, mientras que el grupo experimental ( $72.1 \%$ varones) tuvo una media de edad de 15.91 años. Todos los participantes cumplieron los criterios de inclusión y exclusión, es decir, todos fueron usuarios de la red pública de salud — podían estar adscritos a algún programa salud—, tuvieron una puntuación distinta de 0 en la evaluación con la escala de suicidalidad de Okasha - validada en Chile por Salvo et al. (2009)—, y tenían una edad entre 15 y 19 años; mientras que quedaban excluidos todos aquellos que (a) cursaban un episodio maníaco o psicótico agudo, (b) tuvieran una adicción como problema de salud principal, (c) no tenían capacidades de habla, lectura y visión bien conservadas, y (d) no contaban con familiar o apoderado.

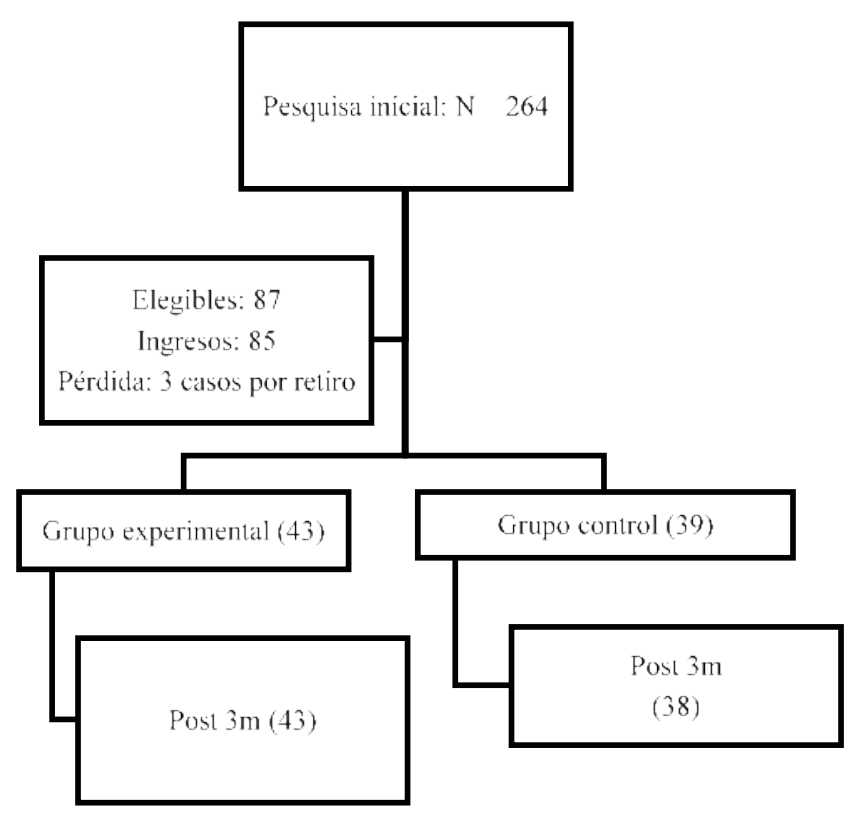

Figura 1.

Flujograma participantes en el estudio 


\section{Instrumentos}

\section{Cuestionario sociodemográfico}

Cuestionario ad hoc para los familiares de los participantes, que incluye datos de salud actual, antecedentes personales y familiares de salud física y mental.

\section{Escala de Suicidalidad de Okasha}

Diseñada por Salvo et al. (2009), es un instrumento de cuatro ítems, con puntuación de 0 a 3 , en la que, entre más puntuación, mayor ideación suicida. La prueba muestra un alfa de Cronbach de.89 en el estudio de adaptación a Chile. En este estudio, la consistencia interna para la escala total fue de .70 .

Este instrumento es el recomendado por el Ministerio de Salud de Chile al momento de inicio del reclutamiento, dada su eficacia para detectar casos graves - que no entran en este estudio-, y por sus adecuadas propiedades para muestras con ideación suicida de baja gravedad y complejidad (MINSAL, 2013, 2017).

\section{Cuestionario de Salud SF-36}

En su versión adaptada en Chile (Olivares, 2006), este instrumento recoge ocho medidas de salud física y mental. Respecto a su fiabilidad, la escala cuenta, en la adaptación de Olivares (2006), con un alfa de Cronbach entre sus escalas que varía entre .82 y .91 . En el presente estudio, se obtuvo un alfa para el total de ítems sobre calidad de vida relacionada con la salud física de .75 , y un coeficiente de consistencia interna de .84 para los ítems sobre la calidad de vida relacionados con la salud mental.

\section{Inventario de Perspectiva Temporal de Zimbardo}

Esta prueba, diseñada originalmente por Zimbardo y Boyd (1999), mide cinco dimensiones temporales: pasado positivo, pasado negativo, presente hedonista, presente fatalista, y futuro. En la adaptación chilena (Oyanadel et al., 2014), la prueba mantuvo su estructura factorial original, y los factores contaron con una confiabilidad que oscila entre un alfa de Cronbach de .59 en pasado positivo y de .80 en pasado negativo. En este estudio, se obtuvieron los siguientes coeficientes de consistencia interna (alfa de Cronbach): pasado negativo $=.73$; pasado positivo $=.62$; presente fatalista $=.68 ;$ presente hedonista $=.75 ; \mathrm{y}$ futuro $=.68$.

Estos índices generales permiten obtener la dimensión compleja desviación del perfil temporal equilibrado — conocida en inglés como deviation from the balanced time perspective (DBTP) - que representa un cálculo donde una menor puntuación indica una mayor concordancia con el perfil equilibrado. Para realizar este cálculo, se sigue la siguiente fórmula —propuesta originalmente por Stolarski et al. (2011) y utilizada con la nomenclatura en español en posteriores estudios clínicos (Oyanadel \& Buela-Casal, 2014)-: $\sqrt{\text { DBTP }=(\text { oPN-ePN })^{2}+(\text { oPP-ePP })^{2}+(\mathrm{oPF}-\mathrm{ePF})^{2}+(\mathrm{oPH}-\mathrm{ePH})^{2}}$

Nota. La medida oPN es la puntuación óptima y esperada para $\mathrm{PN}$, y ePN es la puntuación observada en el participante. Para las otras dimensiones, los valores utilizados como constante son: $\mathrm{oPN}=1.95 ; \mathrm{oPP}=4.60 ; \mathrm{oPF}=1.50 ; \mathrm{oPH}=3.90 ; \mathrm{oF}=4.00$.

\section{Procedimiento}

Inicialmente, para la selección de la muestra se hizo contacto a través de establecimientos educativos, para luego invitar a quienes eran usuarios de la red pública de salud. Luego de consentir su participación en el estudio, se solicitó, en el caso de los menores de 18 años, el consentimiento de un familiar responsable o tutor legal. Posteriormente, los participantes que obtuvieron puntuaciones distintas de 0 en la escala de Okasha fueron invitados a participar en el programa "Serenamente", del que previamente habían tenido conocimiento por la hoja informativa del estudio.

Luego del pretest, los participantes fueron asignados al azar a los dos grupos por un asesor metodológico independiente al grupo de investigación. Tras esta agrupación, los participantes del grupo experimental asistieron a sesiones grupales dirigidas por un equipo compuesto por un psicólogo clínico y un estudiante avanzado en rol de colaborador, siendo ambas personas entrenadas en el programa manualizado.

Específicamente, la intervención del grupo experimental consistió en ocho sesiones semanales del programa "Serenamente" en el Hospital de La Serena, centro de referencia para la provincia participante en el estudio. El programa, además de una sesión inicial y otra de cierre, incluyó dos sesiones dedicadas a trabajar cada dimensión temporal —pasado, presente, futuro- Adicional a las sesiones presenciales, el entrenamiento grupal contó con un disco compacto - Compact Disc o CD, en inglés- con material escrito —disponible en audio y video-, y un cuaderno de trabajo. Todos los participantes tuvieron acceso al sitio web y contacto con el tutor del programa. Además, se resguardó la integridad del tratamiento - con la grabación de las sesiones - y de la evaluación — con una lista de chequeo de cumplimiento-.

\section{Análisis de datos}

Para determinar el tipo de estadístico a utilizar, se llevó a cabo un análisis de la distribución de las variables que se consideraban de validez interna, en este caso, las variables primarias - no transformadas - que evaluaban la perspectiva temporal. Todas ellas ofrecieron coeficientes que apoyan la asunción de distribución normal, pues se obtuvieron las siguientes $\mathrm{Z}$ de Kolmogorov-Smirnov para cada una 
de las dimensiones: pasado negativo $=0.60(p=.860)$; pasado positivo $=0.99(p=.277) ;$ presente fatalista $=0.71$ $(p=.695)$, presente hedonista $=0.65(p=.789)$; $\mathrm{y}$ futuro $=0.87(p=.223)$. Con estos datos, se decidió utilizar análisis paramétricos.

Por otra parte, para los análisis descriptivos se llevaron a cabo análisis de frecuencia y porcentajes; y para determinar la validez interna se tomó como variable dependiente la desviación del perfil temporal equilibrado (DBTP), al ser una medida compuesta por todas las medidas primarias de la perspectiva temporal.

Finalmente, los contrastes intergrupo e intragrupales se llevaron a cabo mediante contrastes de puntuaciones medias t-tests; y el tamaño del efecto fue determinado por la $d$ de Cohen, para lo cual se calculó el porcentaje de cambio.

\section{Resultados}

En primer lugar, y como criterio de validez interna, se quiso conocer si un programa basado en la modificación de la perspectiva temporal lograba modificar esta perspectiva. Para ello, se tomó como medida de resultados el índice de perspectiva temporal compuesto (DBTP).

Como resultados, por medio de un contraste t-test para muestras independientes, no se observaron diferencias significativas pre-intervención en la dimensión DBTP $(t(80)=2.26)$ entre el grupo experimental $-\mathrm{GE}-(M=3.39 ; D T=0.82)$ y el grupo control $-\mathrm{GC}-(M=3.36 ; D T=0.53)$; pero sí se observaron diferencias en la post-intervención en favor del grupo experimental en la dimensión de equilibrio temporal, con un coeficiente de $t(80)=2.71 ; p=.008$ (GE: $M=2.97$, $D T=0.56$; GC: $M=3.35, D T=0.7)$, diferencia que obtuvo un tamaño de efecto moderado $(d=-0.59)$. Estos hallazgos indican que el programa logró mejorar el perfil equilibrado en el grupo tratado con una terapia basada en la perspectiva temporal —el programa "Serenamente"-.

Ahora bien, con el fin de responder al objetivo general en este estudio de reducir la prevalencia de ideación suicida en los participantes del grupo experimental, se llevó a cabo un contraste de medias para muestras relacionadas para el GE y el GC en la medida de ideación suicida, en donde se observó un efecto principal significativo en el paso del tiempo $[t(80)=3.12 ; p=.003)]$, ya que ambos grupos disminuyeron sus puntuaciones. Sin embargo, los contrastes pre y postintervención, separando el grupo control del grupo experimental, mostraron que en el grupo que recibió el programa de tratamiento se disminuyó significativamente la ideación suicida $[t(42)=3.01 ; p=.004]$, con un tamaño del efecto de $d=0.80$, y un porcentaje de reducción de la ideación suicida del $41.3 \%$-disminución que no fue alcanzada por el grupo control $[t(37)=1.41]$ —. La Figura 2 ilustra este efecto diferencial del programa.

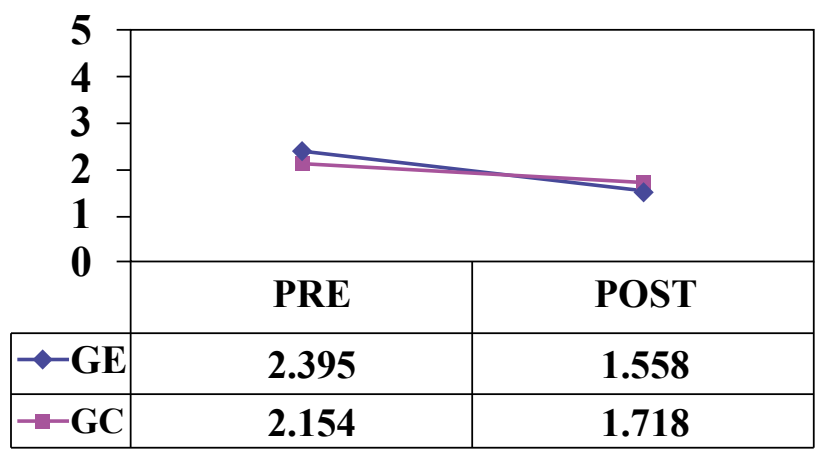

Figura 2.

Cambio en las puntuaciones medias en ideación suicida

Nota. Medición pre y postintervención para los grupos experimental (GE) y control (GC).

Por otra parte, con respecto a los efectos indirectos del programa sobre la calidad de vida, se llevaron a cabo contrastes sobre los datos globales en la escala de calidad de vida en salud física y calidad de vida en salud mental. En la Tabla 1 se resumen los datos descriptivos.

Tabla 1.

Datos descriptivos de dos variables de calidad de vida para el grupo tratado-experimental y el grupo control

\begin{tabular}{ccccc}
\hline Variable & Momento & Grupo & $M$ & $D T$ \\
\hline \multirow{3}{*}{\begin{tabular}{c}
\multirow{2}{*}{ Pre } \\
física
\end{tabular}} & Experimental & 67.24 & 14.91 \\
& & Control & 69.31 & 17.44 \\
& \multirow{2}{*}{ Post } & Experimental & 73.61 & 15.66 \\
& \multirow{2}{*}{ Pre } & Control & 81.90 & 26.69 \\
\hline \multirow{2}{*}{ Calidad salud } & \multirow{2}{*}{ Experimental } & 69.19 & 16.11 \\
mental & Cost & Experimental & 64.59 & 14.29 \\
& & Control & 78.62 & 21.39 \\
\hline
\end{tabular}

Específicamente, respecto a la calidad de vida física, el contraste de medias para muestras relacionadas mostró un efecto principal positivo debido al paso del tiempo, tomando los dos grupos en conjunto $[t(80)=-2.69 ; p=.009)]$; no obstante, en el contraste pre-post por cada grupo por separado, como ocurrió con la dimensión de ideación suicida, ese efecto solo fue significativo para el grupo que recibió el tratamiento $(t(42)=-2.86, p=.005)$, con un $15 \%$ de mejoría en la percepción de calidad de vida relacionada a la salud física $(d=-0.62)$. Finalmente, con respecto a la calidad en salud mental, también se observó un efecto positivo con el paso del tiempo $[t(80)=-2.62 ; p=.011)]$, pero en este caso se produjo un aumento marginalmente significativo en el grupo control $[t(37)=-2.02, p=.047 ; d=-0.61]$. 


\section{Discusión}

Esta investigación ha tenido como propósito principal conocer si un programa basado en la perspectiva del tiempo lograba disminuir la ideación suicida en una población de adolescentes con ideación previa. Con los resultados obtenidos, es posible afirmar que el programa "Serenamente" es efectivo para disminuir la ideación suicida, con una magnitud significativa del tamaño del efecto. De hecho, la mejora en algunos parámetros de la calidad de vida — con efecto medio en la salud física—corroboran esa eficacia.

Los resultados coinciden con lo descrito por algunos autores (Laghi et al., 2009; Oyanadel et al., 2014; Van Beek et al., 2009; Zimbardo et al., 2012) sobre lo promisorio que resulta un programa de perspectiva temporal en la ideación suicida. No obstante, si la perspectiva temporal se asimila mayormente a una característica o faceta de la personalidad, es esperable que para que los cambios sean más permanentes en el tiempo se requieran mayores experiencias para lograr cambios más significativos.

Ahora bien, los cambios que se exhiben tras la intervención en ideación suicida son importantes, debido a que se observa un efecto concomitante en el nivel de calidad de vida en salud física. Sin embargo, con la intervención no se logró mejorar en nivel general de calidad de vida en salud mental.

Estos cambios en la calidad de vida son complejos de explicar, no obstante, en el caso de la calidad de vida física, es posible hipotetizar que las estrategias y juegos basados en ejercicios de mindfulness —utilizados para trabajar la dimensión fatalista del presente- permitieron mejorar la conciencia de la corporalidad; sin embargo, se requiere de nuevos estudios para corroborar esta relación.

Por otra parte, resulta inesperado que el grupo control haya mejorado la calidad de vida en salud mental. Una explicación posible tiene que ver con la expectativa de realizar el tratamiento y estar formando parte de un programa que sería ofrecido a continuación de la evaluación post. También, puede que los grupos no hayan estado equilibrados en alguna comorbilidad no abordada en este estudio, e, incluso, pudo haber habido factores anímicos genéticos que no fueron controlados (Sarmiento-Hernández et al., 2019). No obstante, esta eventual diferencia tiene una significación marginal —al $5 \%$-, un criterio estadístico que empieza a ser cuestionado debido a la concurrencia de errores tipo I (Ioannidis, 2018).

Ahora bien, esta intervención posee varias limitaciones, como: (a) que su efectividad es limitada en el tiempo; (b) que requiere de propuestas futuras que incluyan otros centros, diferentes regiones y equipos; y (c) que hizo falta un seguimiento a largo plazo. Por esto, se debe tener cuidado al momento de extrapolar sus resultados, ya que no se puede saber si la intervención es viable en casos severos, o si los efectos son duraderos, lo que se convierte en una vía de trabajo futura para esclarecer estos aspectos. Adicional a esto, es necesario establecer la aceptabilidad de la intervención mediante una medición específica, o mediante el diseño de un estudio mixto que incorpore elementos cualitativos.

Finalmente, esta investigación aborda el suicidio con una perspectiva incipiente y novedosa, sustentada científicamente, con estrategias versátiles que permitirían una buena aceptabilidad; aspecto considerado clave para disminuir una serie de factores de riesgo en el adolescente que pueden iniciar o fomentar la ideación o posterior intento suicida (Sánchez-García et al., 2018). Por ello, resulta pertinente evaluar su aplicación como programa psicoeducativo para la salud mental del adolescente (Suárez-Colorado et al., 2019).

En conclusión, los resultados apoyan la eficacia de un tratamiento basado en la perspectiva temporal para la disminución de la ideación suicida, además del logro de un perfil temporal equilibrado reconocido como más saludable. Además, el programa muestra mejorías en la calidad de vida relacionada a la salud, por lo que puede constituirse en una herramienta útil y promisoria a nivel preventivo en el contexto de la salud primaria y en el ámbito escolar.

\section{Conflicto de intereses}

Los autores declaran no tener conflicto de intereses para la publicación del presente artículo.

\section{Referencias}

American Psychiatric Association [APA]. (2003). Practice guideline for the assessment and treatment of patients with suicidal behaviors. APA. https://psychiatryonline.org/pb/ assets/raw/sitewide/practice_guidelines/guidelines/suicide. pdf

Asociación Americana de Psiquiatría [APA]. (2013). Guía de Consulta de los criterios diagnósticos del DSM 5. APA.

Avendaño-Prieto, B. L., Betancort-Montesinos, M., BernalAguirre, A., González-Martínez, L. A., Gómez-Sánchez, S. M., \& Villalobos-Sánchez, C. F. (2020). Celos, desesperanza e ideación suicida en población con orientación sexual diversa. Universitas Psychologica, 18(4), 1-12. https://doi. org/10.11144/javeriana.upsy 18-4.cdis

Bahamón-Muñetón, M. J., \& Alarcón-Vásquez, Y. (2018). Diseño y validación de una escala para evaluar el Riesgo Suicida (ERS) en adolescentes colombianos. Universitas Psychologica, 17(4), 1-15. https://doi.org/10.11144/javeriana.upsy17-4.dvee 
Boniwell, I., Osin, E., Linely, P. A., \& Ivanchenko, G. V. (2010). A question of balance: Time perspective and wellbeing in British and Russian samples. Journal of Positive Psychology, 5(1), 24-40. https://doi.org/10.1080/17439760903271181

Bourdon, O., Raymond, C., Marin, M. F., Olivera-Figueroa, L., Lupien, S. J., \& Juster, R. P. (2020). A time to be chronically stressed? Maladaptive time perspectives are associated with allostatic load. Biological Psychology, 152. https://doi. org/10.1016/j.biopsycho.2020.107871

Ceballos-Ospino, G., Suárez-Colorado, Y. P., \& Campo-Arias, A. (2019). Asociación entre matoneo escolar, síntomas depresivos e ideación suicida. CES Psicología, 12(3), 91-104. https://doi.org/10.21615/cesp.12.3.7

Cuijpers, P., de Beurs, D., van Spijker, B., Berking, M., \& Andersson, G. (2013). The effects of psychotherapy for adult depression on suicidality and hopelessness: A systematic review and meta-analysis. Journal of Affective Disorders, 144(3), 183-190. https://doi.org/10.1016/j.jad.2012.06.025

Didonna, F. (2011). Manual Clínico de Mindfulness. Desclée.

Fernandes-Eloi, J., \& Costa Lourenço, J. R. (2019). Suicídio na Velhice - Um Estudo de Revisão Integrativa da Literatura. CES Psicología, 12(1), 80-95. https://doi.org/10.21615/ cesp.12.1.7

Ghahramanlou-Holloway, M., Cox, D. W., \& Greene, F. N. (2012). Post-admission cognitive therapy: A brief intervention for psychiatric inpatients admitted after a suicide attempt. Cognitive and Behavioral Practice, 19(2), 233-244. https://doi.org/10.1016/j.cbpra.2010.11.006

Guille, C., Zhao, Z., Krystal, J., Nichols, B., Brady, K., \& Sen, S. (2015). Web-based cognitive behavioral therapy intervention for the prevention of suicidal ideation in medical interns: a randomized clinical trial. JAMA psychiatry, 72(12), 11921198. https://doi.org/10.1001/jamapsychiatry.2015.1880

Ioannidis, J. P. A. (2018). The proposal to lower $\mathrm{P}$ value thresholds to .005. JAMA, 319(14), 1429-1430. https://doi. org/10.1001/jama.2018.1536

Kinoshita, K., Kinoshita, Y., Shimodera, S., Nishida, A., Inoue, K., Watanabe, N., Oshima, N., Akechi, T., Sasaki, T., Inoue, S., Furukawa, T., \& Okazaki, Y. (2012). Not only body weight perception but also body mass index is relevant to suicidal ideation and self-harming behavior in Japanese adolescents. The Journal of Nervous and Mental Disease, 200(4), 305-309. https://doi.org/10.1097/ NMD.0b013e31824cb29b

LaCroix, J. M., Perera, K. U., Neely, L. L., Grammer, G., Weaver, J., \& Ghahramanlou-Holloway, M. (2018). Pilot trial of post-admission cognitive therapy: inpatient program for suicide prevention. Psychological services, 15(3), 279. https://doi.org/10.1037/ser0000224

Laghi, F., Baiocco, R., D’Alessio, M., \& Gurrieri, G. (2009). Suicidal ideation and time perspective in high school students. European Psychiatry, 24(1), 41-46. https://doi. org/10.1016/j.eurpsy.2008.08.006
Martínez-Baquero, L. C., Vianchá-Pinzón, M. A., Pérez-Prada, M. P., \& Avendaño-Prieto, B. L. (2017). Asociación entre conducta suicida y síntomas de anorexia y bulimia nerviosa en escolares de Boyacá, Colombia. Acta Colombiana de Psicología, 20(2), 178-188. https://doi.org/10.14718/ ACP.2017.20.2.9

Ministerio de Salud de Chile. (2013). Plan Nacional de Prevención del Suicidio, orientaciones para su implementación. MINSAL.

Ministerio de Salud de Chile. (2017). Plan nacional de Salud Mental, 2017-2025. MINSAL.

Montero, I., \& León, O. (2007). A guide for naming research studies in psychology. International Journal of Clinical and Health Psychology, 7(3), 847-862. http://www.redalyc.org/ articulo.oa? $\mathrm{id}=33770318$

Olivares, P. (2006). Estado de Salud de Beneficiarios del Sistema de Salud de Chile, 2004-2005. Superintendencia de Isapres, Departamento de Estudios y Desarrollo.

Olivera-figueroa, L. A., Asthana, S., Odisho, N., Velez, A. L. O., Cuebas, K., \& Cordova, N. M. L. (2016). Emerging Cross-Cultural research: The role of time perspective on well-being, life satisfaction and mindfulness. Advances in Psychology Research. Nova Science Publishers.

Olivera-Figueroa, L. A., Juster, R., Morin-Major, J., Marin, M., \& Lupien, S. (2015). A time to be stressed? Time perspectives and cortisol dynamicsamong healthy adults. Biological Psychology, 111, 90-99. https://doi.org/ 10.1016/j.biopsycho.2015.09.002

Organización Mundial de la Salud [OMS]. (2014). Prevención del suicidio. Un imperativo Global. https://www.who.int/ mental_health/suicide-prevention/es/

Oyanadel, C., \& Buela-Casal, G. (2011). La percepción del tiempo: influencias en la salud física y mental. Universitas Psychologica, 10(1), 149-161. https://doi.org/10.11144/ Javeriana.upsy10-1.ptis

Oyanadel, C., \& Buela-Casal, G. (2014). Percepción del tiempo y psicopatología: Influencia de la orientación temporal en la calidad de vida de los trastornos mentales graves. Actas Españolas de Psiquiatria, 42(3), 99-107. https://actaspsiquiatria.es/repositorio/16/89/ESP/16-89ESP-99-107-549711.pdf

Oyanadel, C., \& Buela-Casal, G. (2017). Percepción del tiempo y salud: La influencia del perfil temporal equilibrado (BTP) y el perfil temporal negativo (NTP) en la salud física y mental. Revista Argentina de Clínica Psicológica, 26(3), 296306. https://doi.org/10.24205/03276716.2017.103

Oyanadel, C., Buela-Casal, G., \& Pérez-Fortis, A. (2014). Propiedades psicométricas del Inventario de Orientación Temporal en una muestra chilena. Terapia Psicológica, 32(1), 57-66. http://dx.doi.org/10.4067/ S0718-48082014000100005

Oyanadel, C., Buela-Casal, G., Araya, T., Olivares, C., \& Vega, H. (2014). Percepción del tiempo: Resultados de una intervención grupal para modificar el 
perfil temporal. Suma Psicológica, 21, 1-7. https://doi. org/10.1016/S0121-4381(14)70001-3

Papastamatelou J., Unger, A., Giotakos, O., \& Athanasiadou, F. (2015). Is Time Perspective a Predictor of Anxiety and Perceived Stress? Some Preliminary Results from Greece. Psychological Studies, 60(4), 468-477. https://doi. org/10.1007/s12646-015-0342-6

Papastamatelou, J., \& Unger, A. (2018). Diferences in Burnout Proneness depend on Time Perspective - Evidence from an Occupational Sample of Industrial Employees and MBAStudents. Dialogues in Clinical Neuroscience \& Mental Health, 1(1), 52-57. https://doi.org/10.26386/obrela.v1i1.8

Pospos, S., Young, I. T., Downs, N., Iglewicz, A., Depp, C., Chen, J. Y., Newton, I., Lee, K., Light, G., \& Zisook, S. (2018). Web-based tools and mobile applications to mitigate burnout, depression, and suicidality among healthcare students and professionals: a systematic review. Academic psychiatry, 42(1), 109-120. https://doi.org/10.1007/ s40596-017-0868-0

Rönnlund, M., Koudriavtseva, A., Germundsjö, L., Eriksson, T., Åström, E., \& Carelli, M. G. (2019). Mindfulness Promotes a More Balanced Time Perspective: Correlational and Intervention-Based Evidence. Mindfulness, 10, 15791591. https://doi.org/10.1007/s12671-019-01113-x

Salvo, L., Melipillan, R., \& Castro, A. (2009). Confiabilidad, validez y punto de corte para la escala de screening de suicidalidad en adolescentes. Revista Chilena de NeuroPsiquiatría, 47(1), 16-23. http://dx.doi.org/10.4067/ S0717-92272009000100003

Sánchez-García, M., Pérez, A., Paino, M., \& Fonseca-Pedrero, E. (2018). Ajuste Emocional y Comportamental en una Muestra de Adolescentes Españoles. Actas Españolas de Psiquiatría, 46(6), 205-216. https://www.actaspsiquiatria. es/repositorio/20/116/ESP/20-116-ESP-205-16-980875.pdf

Sarmiento-Hernández, E., Ulloa-Flores, R., CamarenaMedellín, B., Sanabrais-Jiménez, M., Aguilar-García, A., \& Hernández-Muñoz, S. (2019). Asociación entre el polimorfismo 5-HTTLPR, el intento suicida y la comorbilidad en adolescentes mexicanos con trastorno depresivo mayor. Actas Españolas Psiquiatria, 47(1), 1-6. https:// www.actaspsiquiatria.es/repositorio/21/117/ESP/21-117ESP-1-6-364762.pdf

Seema, R., \& Sircova, A. (2013). Mindfulness-A time perspective? Estonian study. Baltic Journal of Psychology, 14(1), 4-21. https://www.researchgate.net/publica tion/262790161_Mindfulness_-_a_Time_Perspective_ Estonian_Study

Stolarski, M., Bitner, J., \& Zimbardo, P. (2011). Time perspective, emotional intelligence and discounting of delayed awards. Time and Society, 20(3), 346-363. https://doi. org/10.1177/0961463X11414296

Suárez-Colorado, Y., Palacio-Sañudo, J., Caballero-Domínguez, C., \& Pineda-Roa, C. (2019). Adaptación, validez de constructo y confiabilidad de la escala de riesgo suicida Plutchik en adolescentes colombianos. Revista Latinoamericana de Psicología, 51(3), 145-152. http://dx.doi.org/10.14349/ rlp.2019.v51.n3.1

Sword R. M., Sword R. K. M., \& Brunskill S .R. (2015). Time Perspective Therapy: Transforming Zimbardo's Temporal Theory into Clinical Practice. En M. Stolarski, N. Fieulaine \& W. van Beek (eds.), Time Perspective Theory; Review, Research and Application (pp. 481-498). Springer, Cham. https://doi.org/10.1007/978-3-319-07368-2_31

Sword, R. M., Sword, R., Brunskill, S., \& Zimbardo, P. (2014). Time Perspective Therapy: A New Time-Based Metaphor Therapy for PTSD. Journal of Loss and Trauma, 19(3), 197-201. https://doi.org/10.1080/15325024.2013.763632

Turecki, G., \& Brent, D. (2016). Suicide and suicidal behaviour. The Lancet, 387(10024), 1227-1239. https://doi. org/10.1016/S0140-6736(15)00234-2

Van Beek, W., Kerkhof, A., \& Beekman, A. (2009). Future oriented group training for suicidal patients: a randomized clinical trial. BMC Psychiatry. https://doi. org/10.1186/1471-244X-9-65

Ventura-Juncá, R., Carvajal, C., Undurraga, S., Vicuña, P., \& Egaña, J. (2010). Prevalencia de ideación e intento suicida en adolescentes de la Región Metropolitana de Santiago de Chile. Revista Médica de Chile, 138, 309-315. https://doi. org/10.4067/s0034-98872010000300008

Vera-Romero, O., \& Díaz-Vélez, C. (2012). Suicidio en adolescentes de Sudamérica: Un problema creciente. Salud Pública de México, 54(4), 363-364. https://doi.org/10.1590/ S0036-36342012000400003

Yufit, R. I., Benzies, B., Fonte, M. E., \& Fawcett, J. A. (1970). Suicide potential and time perspective. Archives of General Psychiatry, 23(2), 158-163. https://doi.org/10.1001/ archpsyc. 1970.01750020062008

Zimbardo, P., \& Boyd, J. (1999). Putting time in perspective: A valid, reliable, individual-differences metric. Journal of Personality and Social Psychology, 77(6), 1271-1288. https://doi.org/10.1037/0022-3514.77.6.1271

Zimbardo, P., \& Boyd, J. (2009). La paradoja del tiempo. Paidós.

Zimbardo, P., Sword R., \& Sword, R. (2012). The time cure: Overcoming PTSD with the new psychology of time perspective therapy. Jossey-Bass. 